PROCEEDINGS OF THE

AMERICAN MATHEMATICAL SOCIETY

Volume 137, Number 3, March 2009, Pages 1013-1020

S 0002-9939(08)09570-1

Article electronically published on September 24, 2008

\title{
THE LERAY-SCHAUDER CONDITION FOR CONTINUOUS PSEUDO-CONTRACTIVE MAPPINGS
}

\author{
CLAUDIO H. MORALES
}

(Communicated by Nigel J. Kalton)

\begin{abstract}
Over thirty years ago, Kirk raised the question of whether a nonexpansive mapping, defined on a convex domain with nonempty interior, has a fixed point under the Leray-Schauder condition, provided that its domain enjoys the Fixed Point Property with respect to nonexpansive self-mappings. In the present work we have found the answer to this question to be positive, even for a larger class of mappings. The result, indeed, represents a quite significant extension of a large number of theorems obtained in the last forty years. This also includes new theorems for nonexpansive mappings.
\end{abstract}

\section{INTRODUCTION}

Let $X$ be a (real) Banach space. An operator $T: D(T) \subset X \rightarrow X$ is said to be $k$-pseudo-contractive $(k \in \mathbb{R})$ if for each $x, y \in D(A)$ there exists $j \in J(x-y)$ such that

$$
\langle T(x)-T(y), j\rangle \leq k\|x-y\|^{2}
$$

where $J: X \rightarrow 2^{X^{*}}$ is the normalized duality mapping which is defined by

$$
J(u)=\left\{j \in X^{*}:\langle u, j\rangle=\|u\|^{2},\|j\|=\|u\|\right\} .
$$

Here $\langle.,$.$\rangle denotes the generalized duality pairing. For 0<k<1$ in the inequality (1), we say that $T$ is strongly pseudo-contractive, while for $k=1, T$ is simply called pseudo-contractive. It is worth mentioning that this latter class of operators was first introduced by Browder 3. It is an immediate consequence of the HahnBanach Theorem that $J(u)$ is nonempty for each $u$ in $X$. It is also known that $J(u)$ is single-valued if and only if $X$ is smooth.

In addition to generalizing the nonexpansive mappings (mappings $T: D \rightarrow X$ for which $\|T x-T y\| \leq\|x-y\|$ for all $x$ and $y$ in $D$ ), the pseudo-contractive ones are characterized by the fact that $T$ is pseudo-contractive if and only if $I-T$ is accretive (see [2, 8, ).

The Leray-Schauder condition has been extensively used to obtain fixed points of various types of operators for over seventy years. In fact, the Leray-Schauder Principle [13] was originally established for compact mappings defined on a Banach space under the assumption that the set of eigenvectors with eigenvalues greater

Received by the editors January 23, 2008, and, in revised form, March 12, 2008.

2000 Mathematics Subject Classification. Primary 47H10; Secondary 65J15.

Key words and phrases. Pseudo-contractive operators, Leray-Schauder condition, and the fixed point property for nonexpansive self-mappings.

(C)2008 American Mathematical Society Reverts to public domain 28 years from publication 
than one is a bounded set, in order to assure the existence of a fixed point. Today, this condition may be formulated as follows: there exists $z \in \operatorname{int}(D(T))$ such that

$$
T(x)-z \neq \lambda(x-z) \text { for } x \in \partial D(T) \text { and } \lambda>1 .
$$

Since the time of Schauder, many authors have made interesting contributions using this condition. Among them, we mention Browder 2, Petryshyn 21, Nussbaum [19, Webb [26], Reinermann and Schöneberg [23, Reich 22], Schöneberg [25], Gatica and Kirk [7, Kirk [10, Morales [15]. In addition, some compilations may be found in Zeidler [27] and O'Regan and Precup [20].

The main purpose of this paper is to resolve an old question concerning the existence of fixed points for nonexpansive mappings under the Leray-Schauder condition, which was formulated by Kirk (see [10]) over thirty years ago. It is known that the Leray-Schauder condition is weaker than most of the boundary conditions that have been used to derive fixed point theorems. A positive answer to this question has an impact on extending a number of results, as shall be seen throughout the paper. As a matter of fact, we have addressed the question for a much larger family of operators, beyond the nonexpansive ones, where no convexity assumption on the domain of the operator is prescribed.

We say that a mapping $T: D \rightarrow X$ is generalized pseudo-contractive if for each $x \in D$ there exists a number $\alpha(x)<1$ such that

$$
\langle T(x)-T(y), j\rangle \leq \alpha(x)\|x-y\|^{2}
$$

for all $y \in D$ and for some $j \in J(x-y)$. Naturally, generalized contractions (i.e. $\|g(u)-g(v)\| \leq \alpha(u)\|u-v\|$ for all $v$ in the domain of $g$ ) are generalized pseudo-contractions.

It will be derived that if $T$ is a continuous pseudo-contractive mapping which enjoys the Leray-Schauder condition, then $D \subset f_{r}(D)$ for a suitable $r>0$, where $f_{r}=(1+r) I-r T$. This fact, very important in the theory, was earlier obtained by Martin [14] under the weakly inward condition.

Throughout the paper we assume that $X$ is a real Banach space. We shall denote the closure, the interior and the boundary of $D$ by $\bar{D}$, int $(D)$ and $\partial D$ respectively. We shall also use $B(x ; r)$ and $\bar{B}(x ; r)$ to stand for the open ball centered at $x$ with radius $r$ and the corresponding closed ball with the same center and radius.

\section{MAIN RESULT}

We observe that the problem described in the introduction was already known for Hilbert spaces by the year 1965 (see [2]). However, Theorem 2 below would contribute significantly in obtaining numerous generalizations, proven under stronger conditions. Among them, we can see the following:

Theorem B (Browder [4, 1968). Let $X$ be a uniformly convex Banach space and $G$ a closed bounded and convex subset of $X$ with 0 in the interior of $G$. Suppose $T: G \rightarrow X$ is a nonexpansive mapping such that

$$
T(x) \neq \lambda x \text { for } \lambda>1 \text { and } x \in \partial G .
$$

Then $T$ has a fixed point in $G$.

Later, in 1977, Kirk and Schöneberg extended the result to pseudo-contractive mappings by assuming that the operator is continuous and pseudo-contractive in a domain larger than where the Leray-Schauder condition holds. 
Theorem KS (Kirk-Schöneberg [12]). Let $X$ be a uniformly convex Banach space, $D$ a bounded closed convex subset of $X$ with $\operatorname{int}(D) \neq \phi$, and $G$ an open set containing $D$ such that $\operatorname{dist}(D, X-G)>0$. Suppose $T: G \rightarrow X$ is a continuous pseudo-contractive mapping which sends bounded sets into bounded sets and satisfies, for some $z \in \operatorname{int}(D)$,

$$
T(x)-z \neq \lambda(x-z) \text { for } x \in \partial D \text { and } \lambda>1 .
$$

Then $T$ has a fixed point in $D$.

In the following year, Schöneberg [24] extended Browder's original result for nonexpansive mappings to demicontinuous pseudo-contractive ones, where no convexity in the domain is required. However, the result was still done for Hilbert spaces. By 1979, this author had extended Schöneberg's work to $l_{p}$ spaces as can be seen below:

Theorem M (Morales [15]). Let $X$ be a reflexive Banach space which admits a weakly sequentially continuous duality mapping. Let $D$ be a closed bounded subset of $X$, and let $T: \bar{D} \rightarrow X$ be a continuous pseudo-contractive mapping which satisfies, for some $z \in \operatorname{int}(D)$,

$$
T(x)-z \neq \lambda(x-z) \text { for } x \in \partial D \text { and } \lambda>1 .
$$

Then $T$ has a fixed point in $D$.

We now state and prove a new result for pseudo-contractive mappings under a slightly stronger condition than the classical Leray-Schauder condition. We first state a result used in the proof of Theorem 1 below.

Theorem C (Morales [16]). Let D be an open subset of a Banach space X. Let T be a continuous mapping from $\bar{D}$ into $X$ which is locally strongly pseudo-contractive on $D$. Then the following are equivalent:

(i) $T$ has a fixed point in $D$.

(ii) There exists $z \in D$ such that $\|z-T(z)\| \leq\|x-T(x)\|$ for all $x \in \partial D$.

(iii) There exists an open set $G \subset D$ and $z \in G$ such that $T(x)-z \neq \lambda(x-z)$ for $x \in \partial D$ and $\lambda \geq 1$.

Theorem 1. Let $D$ be a bounded open subset of a Banach space $X$, and let $T$ be a continuous mapping from $\bar{D}$ into $X$ which is locally pseudo-contractive on $D$. Suppose there exists $z \in D$ satisfying

$$
T(x)-z \neq \lambda(x-z) \text { for } x \in \partial D \text { and } \lambda \geq 1 .
$$

Then there exists a nonexpansive self-mapping $g$ of a closed ball in $D$, which has the same fixed points as $T$ in $D$.

Proof. For each $t \in(0,1)$, the mapping $t T+(1-t) z$ is locally strongly pseudocontractive on $\mathrm{D}$ and continuous on $\bar{D}$. Since this mapping also satisfies the LeraySchauder condition, then by Theorem $\mathrm{C}$, it has a fixed point in $D$. This means that $t T\left(x_{t}\right)+(1-t) z=x_{t}$ for some $x_{t} \in D$. Since $D$ is bounded, we deduce that $\left\|x_{t}-T x_{t}\right\|=\left(t^{-1}-1\right)\left\|x_{t}-z\right\| \rightarrow 0$ as $t \rightarrow 1^{-}$. Consequently, we conclude that

$$
\inf \{\|x-T x\|: x \in D\}=0 \text {. }
$$

Therefore, we may select two vectors $v, w \in D$ such that $\|w-T w\|<\|v-T v\|$; otherwise $x-T(x)=0$ for all $x \in D$, implying that $T=I$. Hence, we choose 
$g=I$, which is nonexpansive. In addition, since the mapping $x \mapsto\|x-T(x)\|$ is continuous, we may select the above vector $w$ so that $\|w-T(w)\|>0$. Define the set

$$
D_{o}=\{x \in D:\|x-T x\|<\|v-T v\|\} .
$$

Then $w \in D_{o}$ and hence it is a nonempty open subset of $D$ such that

$$
\|w-T(w)\|<\inf \left\{\|x-T(x)\|: x \in \partial D_{o}\right\}=\|v-T(v)\| .
$$

Since $D_{o}$ is bounded, we select $\delta>0$ and $s \in(0,1)$ such that $D_{o} \subset B(w ; \delta)$ and

$$
s(\|w-y\|+\|y-x\|)+\|w-T(w)\|<\|x-T(x)\|
$$

for all $y \in \bar{B}(w ; \delta)$ and for all $x \in \partial D_{o}$. For each $y \in \bar{B}(w ; \delta)$, define $S: \bar{D}_{o} \rightarrow X$ by $S(x)=T(x)+s(y-x)$. Then $S$ is locally strongly pseudo-contractive and satisfies

$$
\begin{aligned}
\|w-S(w)\| & =\|w-T(w)+s(w-y)\| \\
& \leq\|w-T(w)\|+s\|w-y\| \\
& <\|x-T(x)\|-s\|y-x\| \\
& \leq\|x-S(x)\|
\end{aligned}
$$

for all $x \in \partial D_{o}$. Therefore, by Theorem C, $S$ has a fixed point $x$ in $D_{o}$, and hence $y=(1+r) x-r T(x)$ for $r=1 / s$. This implies $\bar{B}(w ; \delta) \subset f_{r}\left(D_{o}\right)$ with $f_{r}=(1+r) I-r T$. In addition, by a simple extension of Proposition 2 of [18] (done for $r=1$ ) to $r>0, f_{r}$ is globally injective on $D_{o}$. Hence $g_{r}=f_{r}^{-1}$ is a locally nonexpansive mapping from $\bar{B}(w ; \delta)$ onto $D_{o}$. Consequently, it is also globally nonexpansive with the same fixed points as $T$.

Remark 1. Under the assumptions of Theorem 1, we have shown that there exists an open subset $D_{o}$ of $D$ and a closed ball $B$ containing $D_{o}$ for which $D_{o} \subset B \subset f_{r}\left(D_{o}\right)$ for some appropriate positive real number $r$. Now we derive, perhaps, the most general fixed point theorem for pseudo-contractive mappings under the well-known Leray-Schauder condition.

Theorem 2. Let $D$ be a bounded open subset of a Banach space $X$, for which the closed unit ball has the Fixed Point Property (F.P.P.) for nonexpansive selfmappings. Let $T$ be a continuous mapping from $\bar{D}$ into $X$ which is locally pseudocontractive on D. Suppose there exists $z \in D$ satisfying

$$
T(x)-z \neq \lambda(x-z) \text { for } x \in \partial D \text { and } \lambda>1 \text {. }
$$

Then $T$ has a fixed point in $\bar{D}$.

Proof. Suppose, without loss of generality, that $T$ has no fixed points on $\partial D$. Then by Theorem 2, there exists a nonexpansive self-mapping $g$ defined in a closed ball in $D$. Hence by the F.P.P. $g$ has a fixed point, and so does $T$.

As a consequence of Theorem 2, we are able to answer Kirk's question (see [11]), even under a slightly more general formulation where no convexity of the domain of $T$ is required.

Corollary 1. Let $D$ be a bounded open subset of a uniformly convex Banach space $X$. Let $T$ be a continuous mapping from $\bar{D}$ into $X$ which is locally pseudocontractive on D. Suppose there exists $z \in D$ satisfying

$$
T(x)-z \neq \lambda(x-z) \text { for } x \in \partial D \text { and } \lambda>1 \text {. }
$$

Then $T$ has a fixed point in $D$. 
Now we are ready to extend Theorem $\mathrm{B}$ of Browder [4, by removing the convexity assumption on $G$, which, in fact, is also a new result.

Corollary 2. Let $X$ be a uniformly convex Banach space and $G$ a closed and bounded subset of $X$ with 0 in the interior of $G$. Suppose $T: G \rightarrow X$ is a locally nonexpansive mapping such that

$$
T(x) \neq \lambda x \text { for } \lambda>1 \text { and } x \in \partial G .
$$

Then $T$ has a fixed point in $G$.

Based upon the classical result of Kirk [9], we may derive a more general corollary which, in particular, extends Corollary 3.2 of Gatica and Kirk [7].

Corollary 3. Let $X$ be a reflexive Banach space with the unit closed ball having the normal structure. Let $D$ be a bounded open subset of $X$ with $0 \in D$. Suppose $T: \bar{D} \rightarrow X$ is a locally nonexpansive mapping such that

$$
T(x) \neq \lambda x \text { for } \lambda>1 \text { and } x \in \partial D \text {. }
$$

Then $T$ has a fixed point in $\bar{D}$.

Next, we obtain a type of Borsuk Theorem with antipodal points on $\partial D$ for pseudo-contractive mappings, which is a slight generalization of Theorem 1 of [17].

Corollary 4. Let X be a Banach space for which the closed unit ball has the F.P.P. for nonexpansive self-mappings, and let $D$ be a symmetric and bounded neighborhood of the origin in $X$. Suppose $T: \bar{D} \rightarrow X$ is a continuous mapping on $\bar{D}$ and locally pseudo-contractive on $D$ such that

$$
T(-x)=-T(x) \text { for } x \in \partial D .
$$

Then $T$ has a fixed point in $D$.

Proof. Let $x \in \partial D$ such that $T(x)=\lambda x$ for some $\lambda>1$. Due to the symmetry of $D$, the segment $\operatorname{seg}[-x, x] \subset \bar{D}$, and hence $T$ is globally pseudo-contractive on $\operatorname{seg}[-x, x]$. Then for some $j \in J(x-(-x))$,

$$
\langle T(x)-T(-x), j\rangle \leq 4\|x\|^{2},
$$

which implies that $\langle\lambda x, j\rangle \leq 2\|x\|^{2}$. Thus $\lambda \leq 1$. Consequently, the Leray-Schauder condition holds, and Theorem 2 completes the proof.

Now we prove a new result for the so-called generalized pseudo-contractive mappings under the Leray-Schauder condition.

Theorem 3. Let $X$ be a reflexive Banach space, let $D$ an open subset of $X$ and let $T: \bar{D} \rightarrow X$ be a continuous and generalized pseudo-contractive mapping. Suppose there exists $z \in D$ such that

$$
T(x)-z \neq \lambda(x-z) \text { for } x \in \partial D \text { and } \lambda>1 .
$$

Then $T$ has a fixed point in $\bar{D}$.

Proof. We first notice that the set

$$
E=\{x \in D: T(x)-z=\lambda(x-z) \text { for some } \lambda>1\}
$$

is bounded. To see this, let $x \in E$ and $j \in J(x-z)$. Then

$$
\langle z+\lambda(x-z)-T(z), j\rangle \leq \alpha(z)\|x-z\|^{2},
$$


implying that

$$
[1-\alpha(z)]\|x-z\| \leq\|z-T(z)\| .
$$

Therefore, $E$ is bounded, and hence we may assume without loss of generality that $D$ is bounded. Since $T$ is a generalized pseudo-contractive mapping, in particular it is pseudo-contractive on $\bar{D}$. This means that the conclusion and the proof of Theorem 1 hold. In addition, for $r>0$, the mapping $f_{r}=(1+r) I-r T$ satisfies

$$
\left\langle f_{r}(x)-f_{r}(y), j\right\rangle \geq[1+r-r \alpha(x)]\|x-y\|^{2}
$$

for all $x, y \in D$. Now, as a consequence of the proof of Theorem 1 there exists an open set $D_{o}$ of $D$ and a closed ball $B$ containing $D_{o}$ such that $B \subset f_{r}\left(D_{o}\right)$ for a suitable $r>0$. Therefore the mapping $g_{r}=f_{r}^{-1}$ maps $B$ into itself. Moreover, $g_{r}$ is a generalized contraction mapping in the sense of Belluce-Kirk [1]. In fact, for each $u \in B$ there exists a unique $x \in D_{o}$ such that $x=g_{r}(u)$ and

$$
\left\|g_{r}(u)-g_{r}(v)\right\| \leq \beta(u)\|u-v\| \quad \text { with } \beta(u)=\frac{1}{1+r-r \alpha\left(g_{r}(u)\right)}<1
$$

for all $v \in B$. On the other hand, $B$ is a closed convex and weakly compact subset of $X$ where $g_{r}$ has diminishing orbital diameters. Hence, by Corollary 2 of [1, $g_{r}$ has a fixed point in $D_{o}$, and consequently so does $T$.

It turns out that there is an oversight in the proof of Theorem 4 of [17. In fact, it is not clear whether the set $K$ is indeed a subset of $\bar{D}$. However, as a consequence of Theorem 3, we correct the oversight and actually obtain a slight extension of that theorem.

Corollary 5. Let $X$ be a reflexive Banach space, let $D$ an open subset of $X$ and let $T: \bar{D} \rightarrow X$ be a generalized contraction. Suppose there exists $z \in D$ such that

$$
T(x)-z \neq \lambda(x-z) \text { for } x \in \partial D \text { and } \lambda>1 .
$$

Then $T$ has a fixed point in $\bar{D}$.

Following [6], we obtain a new result for the so-called strongly pseudo-contractive mappings relative to a set.

Theorem 4. Let $D$ be an open subset of a reflexive Banach space $X$ and let $T$ be a continuous mapping from $\bar{D}$ into $X$. Suppose that for each $x \in D$ and $r>0$, there exists a positive number $\alpha_{r}(x)<1$ such that

$$
\|x-y\| \leq \alpha_{r}(x)\|(1+r)(x-y)-r(T(x)-T(y))\|
$$

for all $y \in D$. If, in addition, $T$ satisfies the Leray-Schauder condition (4), then $T$ has a fixed point.

Proof. By replacing $T(x)$ by $T(x+z)-z$, one may take $z=0$ in (4). Let $r>0$, and let $f_{r}=(1+r) I-r T$. Then, as a consequence of (5), we obtain that for each $x \in \bar{D}$,

$$
\|x-y\| \leq \alpha_{r}(x)\left\|f_{r}(x)-f_{r}(y)\right\|
$$

for all $y \in \bar{D}$. Since $T$, in particular, is pseudo-contractive, Theorem 3 of [5] implies that $G=f_{r}(D)$ is open. Also, since $f_{r}$ is injective and $f_{r}(\bar{D})$ is closed, $f_{r}(\partial D)=\partial f_{r}(D)$. Consequently, $g_{r}=f_{r}^{-1}$, which maps $\bar{G}$ onto $\bar{D}$, is a generalized contraction mapping. In fact, for each $u \in \bar{G}$ there exists a unique $x \in \bar{D}$ such that $x=g_{r}(u)$ and

$$
\left\|g_{r}(u)-g_{r}(v)\right\| \leq \alpha_{r}(u)\|u-v\|
$$


for all $v \in \bar{G}$ with $\alpha_{r}(u)=\alpha_{r}\left(g_{r}(u)\right)$. In addition, $g$ satisfies the Leray-Schauder condition on $\partial G$. To see this, we first prove that $0 \in G$. This means we need to show that there exists $x \in D$ so that $f_{r}(x)=0$. However, $T$ is pseudo-contractive on $\bar{D}$ and hence the operator $\frac{r}{1+r} T$ is continuous and strongly pseudo-contractive, satisfying the Leray-Schauder condition. Therefore, by Theorem C (see also Theorem 1 of [15]), $\frac{r}{1+r} T$ has a unique fixed point, which is obviously in $D$. Hence, $0 \in G$.

Now we prove that $g$ satisfies the Leray-Schauder condition on $\partial G$. To this end, let $y \in \partial G$ such that $g_{r}(y)=\mu y$. Then there exists $x \in \partial D$ so that $y=f_{r}(x)$. Hence

$$
x=\mu((1+r) x-r T(x)),
$$

which implies that $T(x)=\left[1+r^{-1}\left(1-\mu^{-1}\right)\right] x$. Therefore, $\mu \leq 1$. Now, by Corollary 5, $g_{r}$ has a fixed point, which is also a fixed point of $T$.

Remark 2. Theorem 4 represents a significant extension of Theorem 3 of [6]. In fact, the assumption on $T$ of being Lipschitz has been replaced by simply continuity. Also, neither convexity nor boundedness is required on the domain of the operator $T$.

\section{ACKNOWLEDGEMENT}

The author wishes to thank the referee for useful comments that helped improve the writing of this work.

\section{REFERENCES}

[1] L. P. Belluce and W. A. Kirk, "Fixed-point theorems for certain classes of nonexpansive mappings", Proc. Amer. Math. Soc. 20 (1969), 141-146. MR0233341 (38:1663)

[2] F. E. Browder, "Fixed point Theorems for noncompact nonlinear operators in Hilbert spaces", Proc. Nat. Acad. Sci U. S. A. 53 (1965), 1272-1276. MR0178324(31:2582)

[3] F.E. Browder, "Nonlinear mappings of non-expansive and accretive type in Banach spaces", Bull. Amer. Math. Soc. 73 (1967), 875-882. MR0232255(38:581)

[4] F.E. Browder, "Semicontractive and semiaccretive nonlinear mappings in Banach spaces", Bull. Amer. Math. Soc. 74 (1968), 660-665. MR0230179 (37:5742)

[5] K. Deimling, "Zeros of accretive operators", Manuscripta Math., 13 (1974), 365-374. MR0350538 (50:3030)

[6] J. A. Gatica and W. A. Kirk, "Fixed point theorems for Lipschitzian pseudo-contractive mappings", Proc. Amer. Math. Soc. 36 (1972), 111-115. MR0306993 (46:6114)

[7] J. A. Gatica and W. A. Kirk, "Fixed point theorems for contraction mappings with applications to nonexpansive and pseudo-contractive mappings", The Rocky Mountain J. Math. 4 (1974), 69-79. MR0331136 (48:9470)

[8] T. Kato, "Nonlinear semigroups and evolution equations", J. Math. Soc. Japan 19 (1967), 508-520. MR0226230 (37:1820)

[9] W. A. Kirk, "A fixed-point theorem for mappings which do not increase distances", Amer. Math. Monthly 72 (1965), 1004-1006. MR0189009(32:6436)

[10] W. A. Kirk, "Fixed point theorems for nonexpansive mappings satisfying certain boundary conditions", Proc. Amer. Math. Soc. 50 (1975), 143-149. MR0380527 (52:1427)

[11] W. A. Kirk, "On zeros of accretive operators in uniformly convex spaces", Bollettino U. M. I. 17 (1980), 249-253. MR578355 (81g:47061)

[12] W. A. Kirk and R. Schöneberg, "Some results on pseudo-contractive mappings", Pacific J. Math. 71 (1977), 89-100. MR0487615 (58:7234)

[13] J. Leray and J. Schauder, "Topologie et équations fonctionelles", Ann. Sci. École Norm. Sup. 51 (1934), 45-78. MR 1509338

[14] R. H. Martin, "Differential equations on closed subsets of a Banach space", Trans. Amer. Math. Soc. 179 (1973), 399-414. MR0318991 (47:7537) 
[15] C. H. Morales, "Pseudo-contractive mappings and the Leray-Schauder boundary condition", Comment. Math. Univ. Carol. 20 (1979), 745-756. MR555187 (80k:47067)

[16] C. H. Morales, "On the fixed-point theory for local k-pseudocontractions", Proc. Amer. Math. Soc. 81 (1981), 71-74. MR589138(82c:47072)

[17] C. H. Morales, "Remarks on pseudo-contractive mappings", J. Math. Anal. Appl. 87 (1982), 158-164. MR653612 (83g:47060)

[18] C. H. Morales and S. A. Mutangadura, "On the approximation of fixed points for locally pseudo-contractive mappings", Proc. Amer. Math. Soc. 123 (1995), 417-423. MR1216820 (95c:47067)

[19] R. D. Nussbaum, "Degree theory for local condensing maps", J. Math. Anal. Appl. 37 (1972), 741-766. MR0306986 (46:6107)

[20] D. O'Regan and R. Precup, "Theorems of Leray-Schauder type and applications", Series in Mathematical Analysis and Applications, Vol. 3, Gordon and Breach, Amsterdam, 2001. MR1937722 (2004g:47089)

[21] W. V. Petryshyn, "Structure of fixed points sets of k-set contractions", Arch. Rational Mech. Anal. 40 (1971), 312-328. MR0273480 (42:8358)

[22] S. Reich, "A remark on set-valued mappings that satisfy the Leray-Schauder condition", Atti. Accad. Naz. Lincei. 61 (1976), 193-194. MR0477902 (57:17402)

[23] J. Reinermann and R. Schöneberg, "Some results and problems in the fixed point theory for nonexpansive and pseudocontractive mappings in Hilbert spaces", Fixed Point Theory and Applications, Academic Press, 1976. MR0451052 (56:9339)

[24] R. Schöneberg, "Zeros of nonlinear monotone operators in Hilbert spaces", Canad. Math. Bull. 21 (1978), 213-219. MR0500320 (58:17983)

[25] R. Schöneberg, "Leray-Schauder principles for condensing multi-valued mappings in topological linear spaces", Proc. Amer. Math. Soc. 72 (1978), 268-270. MR507320 (81b:47075)

[26] J. R. L. Webb, "A fixed point theorem and applications to functional equations in Banach spaces", Boll. Un. Mat. Ital. 4 (1971), 775-788. MR0377631(51:13802)

[27] E. Zeidler, "Nonlinear functional analysis and its applications", part I, Springer-Verlag, 1986. MR816732 (87f:47083)

Department of Mathematics, University of Alabama in Huntsville, Huntsville, Alabama 35899

E-mail address: morales@math.uah.edu 